\title{
Discussion: Challenges of earth pressure balance tunnelling in weathered granite with boulders
}

\author{
Nan Zhang MSC \\ PhD candidate, Department of Civil Engineering, School of Naval \\ Architecture, Ocean, and Civil Engineering, Shanghai Jiao Tong University, \\ Shanghai, China
}

\section{Shui-Long Shen PhD}

Professor, Key Laboratory of Intelligence Manufacturing Technology (Shantou University), Ministry of Education, and Department of Civil and Environmental Engineering, College of Engineering, Shantou University, Shantou, Guangdong, China; Discipline of Civil and Infrastructure, School of Engineering, Royal Melbourne Institute of Technology, Victoria, Australia (Orcid:0000-0002-5610-7988)

\author{
An-Nan Zhou PhD \\ Associate Professor, Discipline of Civil and Infrastructure, School of \\ Engineering, Royal Melbourne Institute of Technology, Victoria, Australia \\ (Orcid:0000-0001-5209-5169)
}

\section{Hai-Min Lyu PhD}

Post-Doctoral Fellow, State Key Laboratory of Internet of Things for Smart City, University of Macau, Macau, China (Orcid:0000-0002-2641-8099)

J. N. Shirlaw MSc, CEng, MICE

Senior Consultant, Golder Associates (Hong Kong) Ltd, Wan Chai, Hong Kong, China (corresponding author: nshirlaw@golder.com)

\section{Contribution by J. N. Shirlaw}

The authors are to be commended for presenting a detailed, warts and all, case history of earth pressure balance (EPB) tunnelling in mixed ground formed by the weathering of granite (Zhang et al., 2020). Among the other issues brought out in the paper are the following.

- An overall average advance of 1.05 rings per day $(1.89 \mathrm{~m} /$ day $)$ for the 550 ring section, with an average of just 0.67 rings per day $(1.2 \mathrm{~m} /$ day $)$ achieved in zone 8 , which was mostly in mixed ground with a high proportion of granite rock.

- Two sinkholes.

Excluding the sinkholes, a maximum measured settlement at the ground surface of $290 \mathrm{~mm}$.

- Extensive use of pre- and post-grouting to reduce the risk of further sinkholes or excessive settlement and to help fix boulders in position.

- As shown in Figure 17 of the paper, on average $66.7 \%$ of the cycle time per ring was devoted to cutter changes $(28.2 \%)$, grouting from the surface $(26.2 \%)$ and cleaning out the tail shield $(12.3 \%)$.

- Localised problems with severe water inflows at the joints in the segmental lining, ascribed to wash-out of the tail void grout.

Many of these problems are familiar from previous documented cases of EPB tunnelling in mixed ground resulting from the weathering of rock, particularly when the fresh rock is strong or stronger, and crystalline. Babendererde et al. (2005) discuss an EPB-driven tunnel in Portugal in mixed soil and rock grades of weathered granite: there were 'several' collapses and extremely slow progress. Shirlaw et al. (2003) reported four sinkholes and three further major ground losses over EPB tunnel drives in Singapore at soil/rock interfaces within weathered granite. Shirlaw (2016a) recorded average progress rates of less than $1 \mathrm{~m}$ per two working days for an EPB drive in weathered tuff in mixed-face conditions of $85-99 \%$ rock. On the same drive, the disc cutters needed to be changed more than seven times as frequently in 85-99\% rock, where EPB pressure had to be applied to stabilise the saprolite portion of the face, compared with open-mode tunnelling in a full face of rock grades of tuff. So, most of the problems described by the authors are well known and longstanding issues related to EPB tunnelling in mixed ground resulting from the weathering of strong crystalline rock.

One problem identified in the paper that was not recorded in these previous cases was the locally severe water leakage at the joints and bolt pockets in the tunnel lining, as shown in Figures 9(a) and 9(b) of the paper. The authors state that this was due to wash-out of the grout. Wash-out of the tail void grout would result in uneven loading on and distortion of the rings, with steps and lips at the joints. This would be exacerbated by the shove loads applied to the lining. The leakage would be consistent with there being a consequent mismatch between the meeting faces of the gaskets. It is likely that there was damage to some of the segments due to applying shove pressures to deformed rings. A segmental lining that leaked so badly during construction (Figure 9(c))) and suffered local damage would be a major concern for long-term durability.

The tunnelling clearly experienced major problems with ground control when operating in a mixed face of rock and soil grades of weathered granite, and this is consistent with previous experience worldwide of EPB tunnelling in comparable conditions.

In their conclusions the authors recommend:

- drilling of probe holes from the tunnel boring machine (TBM) to fine-tune the understanding of the ground conditions

- real-time monitoring of cutter wear. 
There is also mention of the ground control problems being a result of encountering boulders and using 'unreasonable' operations parameters, but there is no discussion of how this should be rectified.

There are fundamental issues with using a pressurised EPB $\mathrm{TBM}$ in mixed ground derived from the weathering of strong crystalline rock, as documented in previous projects. Shirlaw (2016a) showed how the penetration index, the specific energy and the cutter wear during EPB tunnelling in mixed-face conditions increased dramatically as the proportion of rock increased, particularly once the proportion of strong rock increased to over $50 \%$ of the total face area. As recorded by Shirlaw et al. (2017) and Kwong et al. (2019), the use of slurry TBMs in similar mixed-ground conditions in weathered granite in Hong Kong did not experience similar problems with ground control. With the slurry TBMs, reasonable rates of progress were achieved despite the need to spend significant amounts of time changing cutters due to the abrasive nature of the rock and the saprolite; nor was there need to use grouting to aid the tunnelling, except at break-in and break-out. A comparison of the performance of EPB and slurry TBMs in mixed-face conditions resulting from the weathering of granitic gneiss is provided in the construction of phase I of the Bangalore metro (Kenyon, 2016). The slurry TBMs on the east-west line required approximately one year of construction time less than the EPBs on the north-south line to complete comparable lengths of tunnel; in addition, one of the final drives on the north-south line was carried out by a slurry TBM that had completed work on the east-west line and was brought over to replace the originally planned EPB TBM (Kenyon, 2016). It is reported that, for the next phase of the Bangalore metro, the client specified the use of slurry TBMs, based on the experience of phase I (Wallis, 2018).

The use of slurry shields has become common in Singapore and Hong Kong when it is anticipated that weathered granite including a significant proportion of mixed-face tunnelling will be encountered (Shirlaw, 2016b). If EPB TBMs are used, it is typically recommended that an additional active face support system using pressurised slurry is incorporated into the TBM, as developed for the Metro Do Porto tunnelling (Babendererde et al., 2005). There is no indication in the original paper that such a system was incorporated into the TBM in Shenzhen.

Shirlaw et al. (2017) discuss the basis for the calculations used in Hong Kong to establish target face pressures for slurry TBM operation and during interventions; these calculations are treated as part of the temporary works design and are prepared and independently reviewed before tunnelling. In Hong Kong, such calculations are typically carried out based on the guidelines provided in GEO Report 249 (Golder Associates (HK) Ltd, 2009) for slurry TBMs and GEO Report 298 (HKIE Geotechnical Division, 2014) for EPB TBMs (these two documents were published by the government of the Hong Kong
Special Administrative Region). Reference can also be made to the guidelines issued by the German Tunnelling Committee (DAUB, 2016). The Hong Kong and German guidelines can be contrasted with the process described by Zhang et al. (2020), where it is reported that the face pressure was 'dynamically adjusted during the tunnelling process by the tunnelling engineers and TBM operators'. This dynamic adjustment appears to have been based on the excavated volume, as determined from counting the number of skips of material removed.

In Figure 7 of the paper it is indicated that the typical volume of material excavated was $200 \mathrm{~m}^{3} /$ ring. For a $1.8 \mathrm{~m}$ long ring and an excavated diameter of $9.15 \mathrm{~m}$, the theoretical volume of ground excavated is $118 \mathrm{~m}^{3} /$ ring. However, the volume would increase due to a number of factors, including bulking, the addition of conditioning agent and the volume of any water flowing into the face and around the TBM skin. In Figure 7, the volume excavated appears to be typically $170-180 \mathrm{~m}^{3} /$ ring up to ring 250 , and thereafter around $220 \mathrm{~m}^{3} /$ ring, but fluctuating dramatically with a maximum value of about $360 \mathrm{~m}^{3} / \mathrm{ring}$. This degree of variation is indicative of major loss of ground and poor face control. However, counting skips is an extremely crude measure of the volume of excavated material. Even the more sophisticated methods of measuring excavated material such as belt weighting or belt scanning are still relatively inaccurate. As discussed in GEO Report 298 (HKIE Geotechnical Division, 2014), such systems are important as they can provide an indication that major over-excavation is occurring. However, the critical control for ensuring face stability is the face pressure, which can be measured accurately, rather than the relatively inaccurate volume measurement. The continuous maintenance of adequate face pressure is a proactive measure to provide face stability, whereas volume measurement is reactive. With volume measurement, control measures can only be taken after significant ground loss has already occurred and been identified. The target face pressure should be based on calculation from the known ground and groundwater conditions, as outlined in GEO Report 298 (HKIE Geotechnical Division, 2014) or DAUB (2016), with procedures to adjust the target pressure if warranted by observations during tunnelling (Shirlaw and Boone, 2009).

From the information in Figures 3 and 6 of the paper, it is evident that the face pressure applied was in places less than the water pressure. It is noted in the paper that many of the ground control problems occurred in the mixed ground in zones 6 and 8 , where the greatest face pressure was applied. However, this was also the area where the water pressure was greatest, as the tunnel was on a down grade. In these zones the applied face pressure appears to have been generally less than the water pressure shown in Figure 3. The significant proportion of time devoted to cleaning the tail skin shows that the spoil was very wet and sloppy, and sliding off the conveyor belt. This is consistent with the face pressure being lower than the water pressure - the result would have been seepage of groundwater towards the TBM, destabilising the saprolite and increasing the water content of the spoil. 
A problem discussed in the paper is the effect of the corestone boulders within the weathered rock on the tunnelling. From the description in the paper it appears that the boulders were coming free from the saprolite matrix and damaging the cutting tools by rolling around the head. Occasionally, boulders also clogged the screw conveyor. The TBM was equipped with disc cutters capable of cutting rock. Ideally, the boulders should be cut in situ, which would avoid the problems described in the paper. Boulders will only be cut if the saprolite matrix surrounding them is stable and strong enough to prevent the boulders being plucked from the face by the forces imposed during cutting. As already discussed, the face pressure applied was often inadequate to balance the water pressure and stabilise the saprolite. In particular, the resulting seepage would have destabilised and eroded the saprolite. So, the boulders would have readily come free, consistent with the description in the paper. The often inadequate face pressure used during tunnelling would thus have been a major factor in causing the problems related to the corestone boulders.

Another significant problem discussed in the paper was the wash-out of the tail void grout. Wash-out cannot occur unless there is groundwater flow that transports the grout away from the tail void around the rings. The obvious transport system in this example is from the tail void, along the overcut around the shield skin and into the excavation chamber. This will occur if the face pressure is less than the groundwater pressure and the ground is sufficiently permeable for the pressure difference to result in significant flow. Once this has occurred, there is the potential for

the partially grouted rings to distort, creating extra flow paths by leakage through the joints in the lining, as shown in Figure 9 of the paper

- subsequent grouting into a water-filled tail void, potentially resulting in segregation of the grout and continuing problems with ineffective grouting.

The use of a tail void grout with a $4 \mathrm{~h}$ setting time is likely to have been another factor in the reported wash-out problems.

Based on the evidence presented in the paper, the root causes of many of the problems experienced on this drive were a result of the choice of TBM, the failure to establish the correct operating pressures and inadequate procedures to maintain face stability. These are fundamental issues for tunnelling in the very onerous and abrasive mixed-ground conditions that were identified in the ground investigation prior to tunnelling. Given the demonstrated limitations of the TBM and the operating procedures, the contributor considers it unlikely that refining the knowledge of the ground conditions and cutter wear, as recommended by the authors, would have prevented the problems that occurred during tunnelling.

\section{Authors' reply}

The authors would like to thank the contributor for providing detailed and valuable discussions regarding the article (Zhang et al., 2020). The authors' responses are as follows.

\section{Ground control}

Boulders lowered the advance rate and aggravated the ground disturbance, resulting in difficulties in terms of tunnel face support (Ren et al., 2018a, 2018b). For ground control, the rotation speed of the cutter head should be decreased to reduce the impact in boulder-laden strata. The TBM is recommended to advance with a low penetration rate to minimise the vibration when encountering boulders (Elbaz et al., 2018a, 2018b). Interventions should be conducted with shorter intervals for the timely replacement of disqualified cutting tools, since damaged cutters may accelerate the failure of cutting tools nearby.

In addition to water leakage, machine control is another critical factor influencing lining quality. In full-face rock strata, vibration leads to difficulties in tunnelling along the designed tunnel axis (DTA). Moreover, the trajectory control system of TBMs shows a time lag effect, which hinders the timely adjustment of the pose and trajectory of the machine (Wang et al., 2018). Deviation from the DTA is subsequently beyond the required range. Consequently, this issue brings about difficulties in lining installation and poor-lining quality, which can aggravate water leakage problems. Figure 9(c) of the paper shows a submerged work zone. This was mainly attributed to drainage through grouting holes in the segment (see Figure 18) before construction of the next ring.

\section{Selection of TBM type}

Geological investigations conducted before the excavation showed the presence of both soft layers and hard rock. Therefore, a combined TBM was used for the studied section to adapt to the frequently varying strata. The conventional shield machine, which was designed for tunnelling in soft layers initially, was improved to increase the working performance when encountering hard strata. For example, the stiffness and strength of the cutter wheel were increased to minimise the deformation in the case of direct contact with hard strata. The disc cutters were improved to be capable of high-strength cutting rock. The main bearing and main drive were also improved to provide enough power when encountering rock.

A slurry TBM may have been helpful to increase the tunnelling performance in zones 6 and 8, where heavy over-excavation occurred. The highly weathered granite in both zones remained stable as undisturbed soil, but was extremely easy to disturb by water alternation or ground disturbance. Therefore, a slurry TBM or a pressurised slurry support system would be recommended to maintain the stability of the tunnel face and mitigate ground settlement. However, a slurry TBM requires both water supply and slurry treatment. Since the case reported 

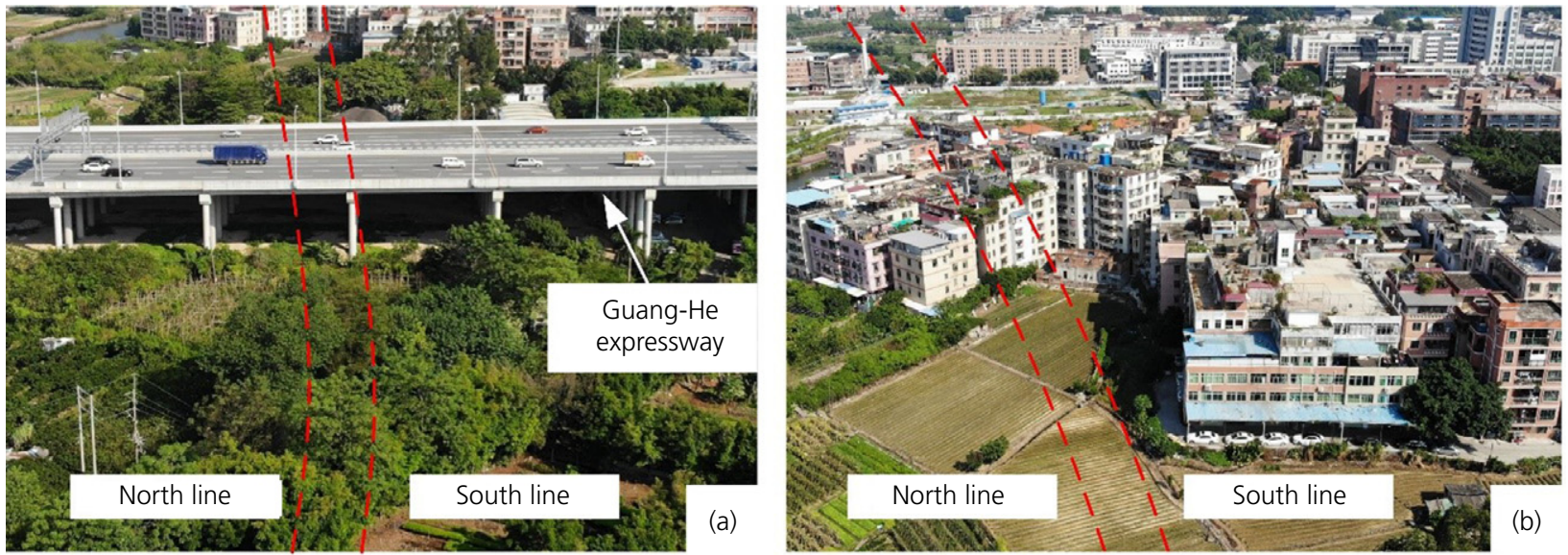

Figure 20. Localised tunnel path through (a) expressway and (b) residential area

in the paper was in the centre of Guangzhou city, it was difficult to arrange a slurry treatment device in this location. Figure 20 shows the localised tunnel path in the urban centre. As mentioned in the paper, some tunnel projects in Shenzhen have employed a slurry-type TBM; for example, a slurry-type TBM is being used on Chunfeng tunnel, under construction.

\section{Tunnel face pressure}

During the tunnelling process of each ring, the thrust cylinder strokes, which indicate the advancing distance of the machine, were carefully monitored by the TBM operators. In general, these strokes should correspond to the volume of the excavated earth estimated by counting skips. Inadequate strokes indicate that over-excavation is probably occurring. In this case, the TBM is ceased and surface grouting is expected to be implemented. Therefore, control measures can be adopted before significant geohazards are observed.

The face pressure setting is very important for maintaining tunnel face stability. As mentioned in the paper, spewing mainly occurred in zone 7 of the full-face rock strata. The advance rate was very low here $(5-10 \mathrm{~mm} / \mathrm{min})$ due to the low penetration rate when cutting rock. The excavated material was mainly a mixture of rock powder and groundwater, which could not be transported by the conveyor belt. As an experiment, the applied face pressure was adjusted up to 3.8 bar $(380 \mathrm{kPa})$, which was higher than the water pressure, by adding air pressure. However, the pressure in the excavation chamber was hard to maintain for the entire tunnelling process, and spewing occurred during pressure relief. Therefore, drainage through grouting holes in the segment was adopted on site before tunnelling the next ring.

The face stability in zones 6 and 8 was very poor. The increase in the applied face pressure subsequently aggravated the formation of mud cakes. This can be attributed to the increase of excavated material in the soil chamber, which further clogged the cutter head opening and resulted in heavy flat wear of the disc cutters on the centre part of the cutter wheel. Accordingly, the authors recommended increases in the dosage of the conditioning agent and the concentration of the foaming agent solution in order to decrease cutter head clogging in the highly weathered granite stratum.

Completely weathered granite is sensitive to ground disturbance and water alternation and shows poor stability for boulders to be cut in position. A high face pressure will stabilise the tunnel face, but will also lead to the formation of mud cakes.

\section{Wash-out problem}

The authors agree with the discusser that the tail void caused by the wash-out of grout resulted in the segment uplifting in the full-face rock strata, which might have led to the ellipticshape deformation of the segments. This aggravated the groundwater leakage through the joints. A modified grout with increased proportions of sand and fly ash could reduce the initial setting time to ease the wash-out problem (Zhang et al., 2018).

\section{Concluding remarks}

A slurry TBM is preferable in mixed-face strata of completely and highly weathered granite owing to its capability of finetuning face pressure, maintaining face stability and reducing ground settlement. However, site conditions and environmental factors become the major concerns for the selection of TBM type. In the project considered in the paper under discussion (Zhang et al., 2020), there was not enough space for slurry treatment equipment (e.g. a mud pond) as the construction site was in an urban centre. In addition, the transportation of slurry is subjected to regulatory limitations during the daytime in the city centre. Large slurry treatment equipment may have also had an 
impact on the surrounding environment, for example ground settlement and ground disturbance ( $\mathrm{Xu}$ and Zong, 2011). Therefore, the EPB method was used for the studied section.

The authors believe that the heavy flat wear occurred owing to cutter head clogging, which can only be inferred from the variation of the operation parameters as mentioned in the paper. Accordingly, it would be great progress if conditions in the excavation chamber during tunnelling could be visualised. This needs the collaborative work of equipment manufacturers, construction site workers and researchers. Meanwhile, the recommendation of real-time monitoring of cutter wear conditions - a part of 'visualised tunnelling' - is favourable in planning the timings of chamber openings to reduce unnecessary cutter inspections, especially in difficult ground conditions.

\section{REFERENCES}

HKIE (Hong Kong Institution of Engineers) Geotechnical Division (2014) Ground Control for EPB TBM Tunnelling. Civil Engineering and Development Department, The Government of the Hong Kong Special Administrative Region, Hong Kong, China, GEO Report 298

Babendererde S, Hoek E, Marinos PG and Cardoso AS (2005) EPB-TBM face support control in the Metro do Porto Project, Portugal. Proceedings of 2005 Rapid Excavation \& Tunneling Conference, Seattle, WA, USA (Hutton JD and Rogstad WD (eds)). Society for Mining, Metallurgy and Exploration, Littleton, $\mathrm{CO}$, USA.

DAUB (Deutscher Ausschuss für unterirdisches Bauen eV) (2016) Recommendations for Face Pressure Support Calculations for Shield Tunnelling in Soft Ground. DAUB, Cologne, Germany.

Elbaz K, Shen SL, Cheng WC and Arulrajah A (2018a) Cutter-disc consumption during earth pressure balance tunnelling in mixed strata. Proceedings of the Institution of Civil Engineers Geotechnical Engineering 171(4): 363-376, https://doi.org/10.1680/ jgeen.17.00117.

Elbaz K, Shen SL, Cheng WC, Arulrajah A and Shirlaw JN (2018b) Discussion: cutter-disc consumption during earth pressure balance tunnelling in mixed strata. Proceedings of the Institution of Civil Engineers - Geotechnical Engineering 171(6): 559-561, https://doi.org/10.1680/jgeen.18.00129.

Golder Associates (HK) Ltd (2009) Ground Control for Slurry TBM Tunnelling. Civil Engineering and Development Department, The Government of the Hong Kong Special Administrative Region, Hong Kong, China, GEO Report 249.

Kenyon P (2016) Getting Bangalore Metro north-south line back on track. TunnelTalk, 20 January. See https://www.tunneltalk.com/ India-25Jan2016-Bangalore-Metro-Phase-I-north-south-line-
construction-troubles-and-Phase-II-preparations.php (accessed $12 / 10 / 2020)$

Kwong AKL, Ng CCW and Schwob A (2019) Control of settlement and volume loss induced by tunneling under recently reclaimed land. Underground Space 4(4): 289-301.

Ren DJ, Shen JS, Chai JC and Zhou A (2018a) Analysis of disk cutter failure in shield tunnelling using 3D circular cutting theory. Engineering Failure Analysis 90: 23-35, https://doi.org/10.1016/j. engfailanal.2018.02.015.

Ren DJ, Shen SL, Arulrajah A and Cheng WC (2018b) Prediction model of TBM disc cutter wear during tunnelling in heterogeneous ground. Rock Mechanics and Rock Engineering 51(11): 3599-3611, https://doi.org/10.1007/s00603-018-1549-3.

Shirlaw JN (2016a) Pressurised TBM tunnelling in mixed face conditions resulting from tropical weathering of igneous rock. Tunnelling and Underground Space Technology 57: 225-240.

Shirlaw JN (2016b) The choice of EPB or slurry shields for tunnelling in mixed ground conditions resulting from tropical weathering. Underground Singapore 2016: 191-207.

Shirlaw JN and Boone S (2009) Geotechnical engineering for TBM tunnelling in soft and mixed ground. Geotechnical Engineering, Journal of the SE Asian Geotechnical Society 40(2): 117-126.

Shirlaw JN, Ong JCW Rosser HB et al. (2003) Local settlements and sinkholes due to EPB tunnelling. Proceedings of the Institution of Civil Engineers - Geotechnical Engineering 56(4): 193-211, https://doi.org/ 10.1680/geng.2003.156.4.193.

Shirlaw JN, Saw AL, Dudouit F and Salisbury D (2017) Operating pressures for twin slurry TBM drives, SCL1103, Hong Kong. Proceedings of the 16th Australasian Tunnelling Conference 2017: Challenging Underground Space: Bigger, Better, More. Engineers Australia, Barton, ACT, Australia.

Wallis (2018) Bangalore Metro bids for Phase II. TunnelTalk, 15 February. See https://www.tunneltalk.com/India-14Feb2018Bangalore-Metro-bids-for-Phase-II-Reach-6.php (accessed 12/10/2020)

Wang LT, Yang X, Gong GF and Du JN (2018) Pose and trajectory control of shield tunneling machine in complicated stratum. Automation in Construction 93: 192-199, https://doi.org/10.1016/ j.autcon.2018.05.020.

Xu ZF and Zong JL (2011) Analysis of impact of large slurry shield construction on environment. Journal of Shanghai Jiaotong University 45(10): 1567-1570, https://doi.org/10.16183/j.cnki.jsjtu. 2011.10.029.

Zhang N, Shen JS, Zhou AN and Arulrajah A (2018) Tunneling induced geohazards in mylonite rock faults with rich groundwater: a case study in Guangzhou. Tunneling and Underground Space Technology 74: 262-272, https://doi.org/ 10.1016/j.tust.2017.12.021

Zhang N, Shen SL, Zhou AN and Lyu HM (2020) Challenges of earth pressure balance tunnelling in weathered granite with boulders. Proceedings of the Institution of Civil Engineers Geotechnical Engineering, https://doi.org/10.1680/ jgeen.20.00030 